\title{
Optimization of Education Management System Based on Web
}

\author{
Tan Jing \\ Urban Vocational College of Sichuan, Sichuan, China \\ tanjingsc@126.com
}

Keywords: web teaching management system; design; implementation; information technology

\begin{abstract}
With the advent of the global information age and the development of computer science, the positive role of information management technology in social life becomes more and more obvious. Computer-aided management has gone from scratch. The course of development is from immaturity to maturity. By referring to the experience of computer-aided teaching management in many foreign countries, education computer-aided management in our country has developed gradually. In terms of schools, the teaching management is a very important but very troublesome thing, if with the combination of information technology and teaching management information system, the efficiency of the school's teaching management can be greatly improved to reduce the data statistics and analysis of data. Teaching management system development method for top-down and modular system development method includes the development and the order for the system analysis, system design, system implementation, system summary and detailed design and realization of online inquiry, and the administrator to realize modify functions, schedule arrangement, login result, etc. of students' information. The system is divided into front desk and back desk. The front desk is direct to the user, conducting various inquiries about teaching; The background is data statistics, management and control.
\end{abstract}

\section{Introduction}

Nowadays, the development of information technology in vigorously, network, computer for people's life and work brings very much convenience [1], but also injected with more force to the development of teaching, making teaching management mode faces change. At the same time, the network causes that the transmission of teaching content does not need to be restricted by time and space, and the development of network teaching management has become the main trend of current teaching development.

Through the investigation, it was found that many school teachers adopted the way of u-disk copy when managing students' homework. Not only is it too expensive, but it can also cause a lot of trouble for job management if the usb drive is lost or damaged [2]. In other schools, teachers have students do paper work and hand it in. Paper exercise books not only occupy the place, but also are not convenient for management. In addition, some teachers ask students to upload their homework to the mailbox and send it to themselves. In this way, although convenient and fast, it is not conducive to the statistics and late maintenance of jobs. According to the above situation, in order to make resources can be Shared, in order to promote teaching management, also must design and build a on job management, resource management and other management for the integration of teaching management system, which is the teaching management system based on WEB. In this way, it can bring more convenience to teachers and students, promote the sharing of teaching resources, and increase the communication between teachers and students.

\section{Design Principles of the System}

The web-based teaching management system must follow these basic principles when designing. First, standardization. That is to say, when designing, we must comply with the relevant national regulations, school regulations, and industry standards. The unified data format is adopted to describe the standardization and standardization of different business work. Second, modularity [3]. 
The whole teaching management system is divided into several different modules according to functions, which makes the design of the system more convenient and also facilitates maintenance and upgrading. Third, advancement. The design is carried out with the very advanced and mature development mode, which makes the developed educational management system powerful, good performance and has certain stability. Third, security. Security is the main principle for the development and design of the educational administration system, because nowadays the network environment is complex, and it is easy to encounter risks and unsafe factors, causing problems for the use of the system. To do this, all user logins need to be validated and permission is restricted. The log-in password is encrypted with MD5 algorithm. Fourth, interactivity. The interface is simple and easy to operate, so as to enable users to better master and use the system on the basis of guaranteeing the overall function realization of the system.

\section{Function Module Design of the System}

The system architecture is adopted by the B/S, with the help of "model-view-controller", in the form of an application's input, processing, output, process according to the View, the Model, the Controller in the form of separation, thus making an application is divided into a Model layer, control layer and View layer [4]. Moreover, this system is developed based on PHP language and adopts the MVC mode based think Ph P framework. The database system USES mysql. In order to make the overall view layer more effective, the front-end framework of the easy UI is adopted.

This system is divided into three modules from the user role: administrator module, teacher module and student module. The administrator module, the module is mainly provides the administrator in this system that can perform various functions, including user information management, class information management, curriculum information management, class information management and schedule management. The management of user information includes the addition, modification and query of administrator information, the addition, modification and query of teacher information, as well as the addition, modification and query of student information. Class information management includes adding, modifying, and querying classes [4]. Course information table management includes course addition, modification and query [5]. Classroom information management includes adding, modifying, and querying classrooms to maintain classroom resources. The curriculum information management includes the addition, modification, query and printing of the curriculum forms, which can be divided into the query of class schedules, the query of teachers' schedules and the query of classroom schedules. After entering the system as an administrator, you can manage administrator information, class information, student information, teacher information, classroom information, course information and schedule information. Teacher module, the module is mainly provides the teachers in this system that can perform various functions, including the class schedule of query print, classroom schedule query print, teacher's schedule query printing, query and modify their own password of the classroom. After teachers log in the system, they can query, add or modify the curriculum form and teaching room. The student module mainly provides various functions that students can carry out in the system, including query and printing of class schedule, classroom query and modification of their own secret codes.

Its overall functional structure is as follows:

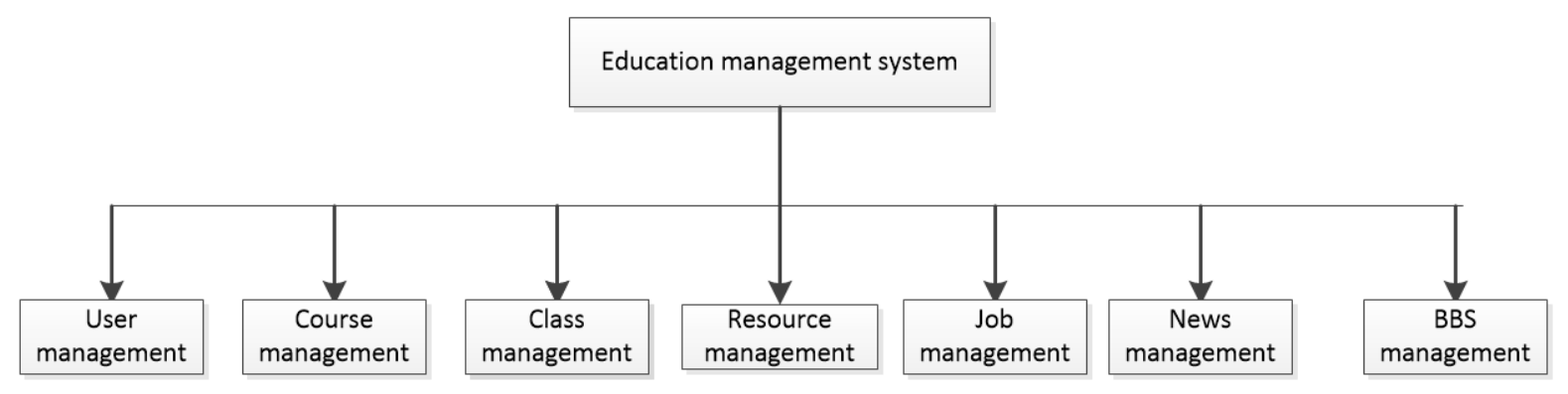

Figure 1: The overall functional structure of the system 
(1) user management

The first is permission management: through permission management, you can add, modify, and delete roles in the system. You can also maintain permissions and scope of use for different roles[6]. Second, user information management: the administrator in the system has the right to add, change and delete users' basic information, authority, identity information, account information, etc. In addition, each individual user in the system has the right to modify his or her personal basic information, such as age, name, date of birth and other basic information.

(2) course management

The first is to manage the course classification, such as dividing and maintaining the course category, or adding, deleting and modifying the course category. The second is to manage the course subjects and maintain them according to the courses of different subjects, such as adding, deleting, or changing or replacing the course subjects.

(3) class management

In this system, there are two kinds of class management, one is term management, the other is teaching class management. So the term class refers to the actual physics class. Because of the factors of teaching resources, it is impossible to carry out teaching activities separately for each period class, so at least one period class constitutes a teaching class, so as to complete teaching tasks. The shift management function in the system refers to the maintenance of the shift, such as adding, deleting and modifying the shift information. The management of teaching classes refers to the addition, deletion or modification of teaching classes on the basis of periodic management.

(4) resource management

The resource management of the system includes two kinds: classified management of teaching resources and list management of teaching resources. The first kind of classified management of teaching resources mainly refers to the addition, modification and deletion of teaching resources. The list management of teaching resources refers to the way in which teaching resources can be added, modified or deleted according to the category of resources and materials. The system can support the uploading of teaching resource files in various formats, such as JPG format, MP4 format, PDF format, rar format, GIF format, zip format, RMVB format, caj format, etc. Teachers can also download and use teaching resources after viewing them.

(5) news management

News management also includes two types, one is news classification management, the other is news list management. News classification management includes the maintenance and management of news categories, as well as the function of adding, deleting and modifying news. News list management includes news addition, deletion, and modification, as well as news placement, news content template maintenance and so on.

(6) BBS management

BBS management refers to the section of the school BBS, say, return card, post and so on a series of access for operation and management, at the same time that the teachers and students have a published their own insights platform, to strengthen the exchange and communication between teachers and students, and to understand students' ideas.

(7) user rights management

There are three types of users in this system, namely students, teachers, administrators and administrators. The permissions of these users are set as follows: all users have password modification permissions of the system management subsystem, information management subsystem view news, BBS communication system permissions. The administrator has all the authority of the system management subsystem and the information management subsystem. The academic administrator can check the personnel in the system management subsystem, and the academic administrator also has all the authority of the system. In addition, the academic staff can delete, modify and add relevant information in the information system.

Teachers have system management subsystem of view permissions, you can view the students' registration, record and also can login the job management system, to release, correcting homework, delete, and so on. In addition, teachers can also enter the score management system to enter and 
query the score. Or delete, modify and add information in the information system.

Students have the function to check courses, assignments and grades. In addition to submitting homework and releasing information in this system, they can also delete and modify the information they publish.

(8) operation management

In the whole teaching management system, the most complex and the most important one is homework management. The design of this module is very difficult. The function of this module is to manage jobs, such as assigning homework, handing in homework, correcting homework, checking homework, communicating and so on.

The first is to arrange the homework, the teacher will assign the homework to be assigned, and the form of the above attachments will be put into the system. This system can upload multiple lattice jobs[7], such as JPG format, MP4 format, PDF format, rar format, GIF format, zip format, RMVB format, caj format, and so on. In addition, teachers can also choose a part of teaching resources for students' reference and use. In addition, when assigning homework, the deadline for homework should be set in the system. After this time, the system will not receive the homework submitted by students.

After the second is to submit homework, the teacher assignments, students finish the homework, before operation by the end of time, in the form of attachment upload homework to system, the format of the assignment system must be able to compatible format.

The third is to revise the work. After receiving the work submitted by the students, the teacher needs to review and revise the work submitted by the students. Through this system, teachers can not only download students' homework in batches, but also check and comment on some students' homework independently, or directly import students' achievements. In addition, teachers can input some comments or marks when correcting homework.

Is the fourth message platform, if students are for the teachers' comments, correcting the homework is in doubt, or have other questions, also can leave a message through the message platform for teachers, and teachers to communicate. And the teacher opens the system, can carry on certain reply to the student's message, very convenient.

The characteristics of the system

1) relatively complete functions

According to the needs of teaching, this system provides some very practical functions, such as course selection management, homework management and performance management. Therefore, it can meet the needs of teachers and students to a certain extent, bring convenience to everyone and promote the smooth teaching.

2) simple and simple interface

The interface of this system is simple and simple, very unified, and the menu function is very complete. After clicking the system icon, teachers and students can quickly enter the operation interface. And there is no need for too much manual input, complete confirmation and error information.

3) convenient and simple operation

The mode adopted by this system is B/S type. The front-end of the system only needs to use a unified browser, which is easy to set up and easy to operate. The system does not require additional training of academic staff, thus reducing the cost of training. In addition, this system does not need to develop and maintain the front-end software, which reduces the investment of the client side, reduces the workload of system maintenance and makes the system easier to expand.

4) more explicit user rights

In the system, users at different levels have different permissions and responsibilities, and can use different functions. Therefore, the system clearly limits the functional modules that each user can use and the data that can be accessed. Only release information BBS, such as students access to courses, upload the homework, but teachers and administrators have educational administration subsystem, management subsystem, achievement management system, this is for the convenience of management. System data privacy protection encryption 


\subsection{Data segmentation}

In the encryption process of different files, in order to facilitate operation, it is necessary to divide the file data into data segments of the same length according to certain rules and arrange them in the form of matrix [8]. In the algorithm designed in this paper, the size of the intercepting data segment is $64 \mathrm{~KB}$, and the resulting matrix is a 256 -order square matrix. The test meets the requirements of the encryption algorithm. In the last data segment, it is usually less than $64 \mathrm{~KB}$. At this point, the zeroing operation is required at the end of the data segment. After decryption, the original data can be obtained by deleting the zeros.

\subsection{Disorderly arrangement}

In the encryption algorithm proposed in this paper, with the method of matrix transformation to disturb the original data, and the transformation matrix in the role of here is equivalent to an encryption key, makes the original data from plain code into a password. After receiving the data, the data can be restored and the original file information can be obtained through the inverse transformation operation of the matrix. Let the data matrix to be processed be:

$$
M=\left[\begin{array}{cccc}
a[1][1] & a[1][2] & \ldots & a[1][j] \\
a[2][1] & a[2][2] & \ldots & a[2][j] \\
\ldots & \ldots & \ldots & \ldots \\
a[i][1] & a[i][2] & \ldots & a[i][j]
\end{array}\right]
$$

$i=1,2, \ldots, k, j=1,2, \ldots, k$

The common matrix transformation is divided into three types: folding transformation, cyclic shift transformation and rotation transformation. In this algorithm, three methods are used to combine, so as to fully scramble the matrix data and achieve high confidentiality. The change process is divided into three steps:

(1) to disturb the data fully, randomly select horizontal or vertical fold in transformation scheme, and generate the corresponding encryption parameters, the level folds down to 0 , vertical fold down to 1 , in order to decrypt.

(2) the function $f$ is used to rotate the matrix to obtain $M_{2}=c[i][j]$. The function $f$ is a simple function that takes $i$ or $j$ as its independent variable. The value range is $1,2, \ldots, k$. For the selection of function AAA, this paper adopts the form of AAA or $f=j+m$. When the circular shift is carried out in the horizontal direction, the $i$ line element is divided horizontally according to the $f$ limit, and the following results can be obtained:

$$
D[i][j]=\left\{\begin{array}{cc}
c[i][k-f+j] & 1 \leq j \leq f \\
c[i][j-f] & f<j<k
\end{array}\right.
$$

When circular displacement is carried out in the vertical direction, the above transformation is similarly divided according to the following formula:

$$
D[i][j]=\left\{\begin{array}{cc}
c[k-f+i][j] & 1 \leq i \leq f \\
c[i-f][j] & f<i<k
\end{array}\right.
$$

Horizontal and vertical transformations are also carried out by random operations. Horizontal operations are denoted as 0 and vertical operations as 1 , and corresponding encryption parameters are generated for use in decryption.

(3) rotate the matrix clockwise or counterclockwise.

\section{Summary and Experience}

The design and implementation of web-based teaching management system promote better 
teaching activities and bring more convenience to teachers and students. Moreover, the system will be constantly improved according to the actual situation of teaching. It is believed that in the future, network teaching management system will become an important trend of education development.

\section{References}

[1] Li X Y, Du D J. Design of and Research on the Web-Based Teaching Management System[J]. Advanced Materials Research, 2013, 760-762(2):977-980.

[2] Jiang Y, Huang Z, Huang Z. Design and Implementation of a General Web-based Course Teaching Management System[C]// Third International Workshop on Education Technology and Computer Science. IEEE Computer Society, 2011:221-224.

[3] Li W J, Shi S S. Research on Semantic Web-Based Teaching Resources Management System[J]. Advanced Materials Research, 2011, 204-210:1366-1369.

[4] Sendari S, Lestari D, Rahmawati Y, et al. Developing prototype of web-based home controlling for teaching trainer[C]// Region 10 Symposium. IEEE, 2016:99-103.

[5] Yao N. Design and Implementation of English Teaching Management System Based on Web[C]// International Conference on Intelligent Transportation, Big Data \& Smart City. IEEE Computer Society, 2018:472-475.

[6] Zhang Y. Design and Development of WEB-based Remote Network Physical Education Teaching Platform in Colleges and Universities[J]. International Journal of Emerging Technologies in Learning, 2018, 13(4):150.

[7] Zgodavova K, Kisela M, Sutoova A. Intelligent approaches for an organisation's management system change[J]. Tqm Journal, 2016, 28(5):760-773.

[8] Jiaqing L V, Liu L, Yun H E, et al. The design of network streaming media system based on college class-teaching[J]. Electronics World, 2016. 